\title{
CircHIPK3/miR-876-5p/PIK3R1 axis regulates regulation proliferation, migration, invasion, and glutaminolysis in gastric cancer cells
}

Qingchun Li', Yuan Tian², Yun Liang ${ }^{2}$ and Chang $\mathrm{Li}^{1 *}$ (D)

\begin{abstract}
Background: Circular RNAs (circRNAs) are a new group of non-coding RNAs that play vital roles in cancer occurrence, including gastric cancer (GC). Nevertheless, the role and underlying regulatory mechanisms of circHIPK3 in GC remain unclear.

Methods: The expression levels of circHIPK3, miR-876-5p, and phosphoinositide-3-kinase regulatory subunit 1 (PIK3R1) were estimated by real-time quantitative polymerase chain reaction (RT-qPCR) assay. The proliferation, migration, and invasion of GC cells were determined by 3-(4, 5-dimethylthiazol-2-yl)-2, 5-diphenyl-2H-tetrazol-3-ium bromide (MTT) and transwell assay. Glutaminolysis of GC cells was assessed by measuring glutamine, glutamate, and a-ketoglutarate levels. The western blot was employed to examine the related-protein expression. The association between miR-876-5p and circHIPK3 or PIK3R1 was predicted and affirmed by bioinformatics database starBase v2.0 and dual-luciferase reporter assay, respectively. Eventually, the xenograft experiment was used to assess the role of circHIPK3 silencing in vivo.
\end{abstract}

Results: CircHIPK3 was upregulated in GC tissues and cells compared with controls, and circHIPK3 was more resistance to RNase $R$ than linear homeodomain interacting protein kinase 3 (HIPK3) mRNA. Silencing of circHIPK3 inhibited GC cells proliferation, migration, invasion, and glutaminolysis as well as tumor tumorigenic ability. Moreover, we also found that miR-876-5p, interacted with PIK3R1, was a target gene of circHIPK3. CircHIPK3 silencing induced effects on GC cells were abolished by silencing of miR-876-5p. In addition, upregulation of PIK3R1 inversed miR-876-5p overexpression-induced effects on GC cells.

Conclusion: The circHIPK3 mediated the proliferation, migration, invasion, and glutaminolysis of GC cells partly through regulation of miR-876-5p/PIK3R1 axis by the mechanism of competing endogenous RNAs (ceRNA), indicating circHIPK3 was a GC-associated circRNA that promoted GC development.

Keywords: CircRNA, ceRNA, Tumorigenic, Target

\footnotetext{
*Correspondence: jewjjx@163.com

1 Department of Gastrointestinal Colorectal and Anal Surgery, China-

Japan Union Hospital of Jilin University, No. 126, Xiantai Street, Changchun 130031, Jilin, China

Full list of author information is available at the end of the article
}

\section{Highlights}

1. CircHIPK3 is obviously overexpressed in gastric cancer tissues and cells.

2. Knockdown of circHIPK3 inhibits gastric cancer cells proliferation, migration, invasion, and glutaminolysis through miR-876-5p/PIK3R1 axis.

3. CircHIPK3 increases PIK3R1 expression by targeting miR-876-5p.

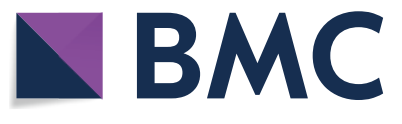

(c) The Author(s) 2020. This article is licensed under a Creative Commons Attribution 4.0 International License, which permits use, sharing, adaptation, distribution and reproduction in any medium or format, as long as you give appropriate credit to the original author(s) and the source, provide a link to the Creative Commons licence, and indicate if changes were made. The images or other third party material in this article are included in the article's Creative Commons licence, unless indicated otherwise in a credit line to the material. If material is not included in the article's Creative Commons licence and your intended use is not permitted by statutory regulation or exceeds the permitted use, you will need to obtain permission directly from the copyright holder. To view a copy of this licence, visit http://creativeco mmons.org/licenses/by/4.0/. The Creative Commons Public Domain Dedication waiver (http://creativecommons.org/publicdomain/ zero/1.0/) applies to the data made available in this article, unless otherwise stated in a credit line to the data. 


\section{Background}

Gastric cancer (GC) is universal malignant tumor all over the world, ranking as the third leading reason of cancerassociated mortality [1]. According to statistics, there are 1,000,000 new cases and 783,000 mortalities of GC in 2018 [2]. The clinical outcomes of GC patients remain poor in most countries, although many advancements have been achieved in terms of technologic methods. Therefore, it is required to discover new diagnosis biomarkers and comprehend the pathophysiology of GC.

Circular RNAs (circRNAs) are a class of circularly configured RNA molecules, lacking $5^{\prime}$ to $3^{\prime}$ polar or polyadenylation tails [3]. Recent data showed that circRNAs were widely expressed in eukaryotes and could act as key regulators in multiple biological processes [4]. Coincidentally, numerous studies revealed that circRNAs were closely associated with the occurrence and progress of malignant tumors, including GC. For example, Rong et al. revealed that circPSMC3 was closely connected with the progression of GC by interacting miRNA-296-5p, indicating that circPSMC3 was novel a target for the therapy of GC [5]. In view of this, it is meaningful to excavate the relevant molecular mechanisms of circHIPK3 in GC. CircHIPK3 (hsa_circ_0000284) is derived from the homeodomain interacting protein kinase 3 (HIPK3) gene and located on chr11 (33307958-33309057). Evidence indicated that circHIPK3 facilitated colorectal cancer cells proliferation and metastasis [6]. In addition, circHIPK3 also was overexpressed in epithelial ovarian cancer, which was associated with poor prognosis of patients [7]. Nevertheless, it was uncertain whether circHIPK3 is associated with regulation of GC development.

Previously published studies have described that aberrant expression of miRNAs played vital role in tumorigenesis, drug-resistance, and immune response [8-10]. By complementary base pairing with the 3 'untranslated region (UTR) of mRNA, miRNA triggered mRNA degradation or translational repression [11]. Furthermore, the tumor inhibition impacts of miR-876-5p were confirmed in many types of tumor cells, including lung cancer [12], hepatocellular carcinoma [13], and GC [14]. An extensive understanding of the function of miR-876-5p in GC was necessary.

Phosphoinositide-3-kinase (PI3K) regulatory subunit 1 (PIK3R1) was identified as a regulator of PI3K/protein kinase $B(A K T)$ signal pathway that was important and complicated in tumorigenesis [15]. Furthermore, PIK3R1 was overexpressed in endometrial cancer cells, and upregulation of miR-495 impeded endometrial cancer cells proliferation while induced apoptosis by directly targeting PIK3R1. The further investigation of the molecular mechanisms of PIK3R1 in GC was required.

Currently, the study was aimed to explore the biological function and underlying mechanism of circHIPK3 in GC. We measured circHIPK3 expression in GC tissues samples and cells. Additionally, functional experiments were used to investigate the regulatory mechanisms of circHIPK3 in regulation proliferation, migration, invasion, and glutaminolysis in GC cells.

\section{Materials and methods}

\section{Tissues collection}

In total of $26 \mathrm{GC}$ patients who had not received any preoperative treatments were registered in the present study. The GC tissues and contiguous noncancerous tissue samples were harvested from patients with surgery at China-Japan Union Hospital of Jilin University and then transferred to $\mathrm{a}-80^{\circ} \mathrm{C}$ refrigerator for further preservation until further use. All patients offered the written informed consents, and the research was permitted by the Ethics Committee of China-Japan Union Hospital of Jilin University.

\section{Cell culture}

The human normal gastric epithelial cell line GES-1 and GC cells (HGC-27) were bought from China Life Science Academy (Shanghai, China). GC cells (AGS) were gained from the American Type Culture Collection (Rockville, MD, USA). Cells were cultivated in RPMI 1640 medium supplemented with penicillin $(100 \mathrm{U} / \mathrm{mL})$, streptomycin $(100 \mathrm{mg} / \mathrm{mL})$, and $10 \%$ fetal bovine serum (GIBCO BRL, Grand Island, NY, USA) in a suitable environment containing $5 \% \mathrm{CO}_{2}$ at $37^{\circ} \mathrm{C}$.

\section{Real-time quantitative polymerase chain reaction (RT-qPCR)}

RNA samples were isolated from GC tissues and cells using Trizol reagent (Takara, Dalian, China) in accordance with the manufacturer's instructions. The complementary DNA was synthesized using $2 \mu \mathrm{g}$ of RNA template as template and reverse transcription kit (Takara) or microRNA Reverse Transcription Kit (Qiagen, Hilden, Germany). All complementary DNA products were quantitatively analyzed using SYBR Green Real-Time PCR Master Mix (Qiagen) under ABI Prism 7900 real-time PCR system (Applied Biosystems, Foster City, CA, USA). The transcription levels of circHIPK3, HIPK3, miR-876-5p, and PIK3R1 were computed based on the $2^{-\Delta \Delta \mathrm{Ct}}$ method. Notable, phosphate dehydrogenase (GAPDH) and small nuclear RNA U6 were used to as housekeeping genes.

The sequences of primers were listed:

circHIPK3 (F-5'-GGGTCGGCCAGTCATGTATC-3'; R-5'-ACACAACTGCTTGGCTCTACT-3');

HIPK3 (F-5'-TCACAAGTCTTGGTCTACCCA-3'; R-5'-CACATAGGTCCGTGGATAGTTTC-3'); miR-876-5p (F-5'-GCCGAGTGGATTTCTTTGTG-3'; R-5'-CTCAACTGGTGTCGTGGA-3'); PIK3R1 (F-5'-ACCACTACCGGAATGAATCTCT-3'; R-5'-GGGATGTGCGGGTATATTCTTC-3'); 
GAPDH (F-5'-TCCCATCACCATCTTCCAGG-3'; R-5'-GATGACCCTTTTGGCTCCC-3');

U6 (F-5'-AACGCTTCACGAATTTGCGT-3'; R-5'CTCGCTTCGGCAGCACA-3').

\section{Transfection assay}

Specific small interfering RNA (siRNA) against circHIPK3 (si-circHIPK3) and siRNA scrambled control (si-NC), and short hairpin RNA objecting circHIPK3 (shcircHIPK3) and shRNA scrambled control (sh-NC) were purchased from GenePharma (Shanghai, China). The miR-876-5p mimic (miR-876-5p) and its negative control (miR-NC), and miR-876-5p inhibitor (anti-miR-876-5p) and its negative control (anti-miR-NC) were designed and acquired from Thermo Fisher Scientific (Waltham, MA, USA). HGC-27 and AGS cells were transfected with plasmids or oligonucleotides using Lipofecamine2000 (Invitrogen, Carlsbad, CA, USA). The sequences for sicircHIPK3, si-NC, sh-circHIPK3 and sh-NC were as follows: si-circHIPK3: 5'-CUACAGGUAUGGCCUCAC A-3', si-NC: 5'-UUCUCCGAACGUGUCACGUTT-3', sh-circHIPK3: $\quad 5^{\prime}$-ccggCUACAGGUAUGGCCUCAC AttcaagagaTGTGAGGCCATACCTGTAGTTTTTTGG TACC-3', sh-NC: 5'- ccggUUCUCCGAACGUGUC ACGUTTttcaagagaAATCGTGACACGTTCGGAGAA TTTTTTGGTACC-3'. For overexpression of PIK3R1 (PIK3R1), the primers were used for amplification and then cloned in the mammalian expression pcDNA3.1 vector (Invitrogen), PIK3R1 F-5'-CCGGAATTCATG AGTGCTGAGGGGTACCAGTAC-3'; and R-5'-CCG CTCGAGATCGCCTCTGCTGTGCATATATA- 3 '.

\section{RNase $\mathrm{R}$ treatment}

$3 \mathrm{U} / \mathrm{mg}$ of RNase R (Epicenter, Madison, WI, USA) was applied to treat RNA for $15 \mathrm{~min}$ at $37^{\circ} \mathrm{C}$, using Mock as control.

\section{Cell proliferation assay}

HGC-27 and AGS cells were seeded into the 96-well plates with a concentration of 3000 cells per well and incubated overnight. After transfection, $20 \mu \mathrm{L}$ of 3-(4, 5-dimethylthiazol-2-yl)-2, 5-diphenyl-2H-tetrazol-3-ium bromide (MTT; Beyotime, Shanghai, China) was added into the 96-well plates at the designated time points. After further culturing for $4 \mathrm{~h}$, the supernatants were replaced with $150 \mu \mathrm{L}$ of dimethyl sulfoxide (DMSO). The cell viability was assessed by detecting optical density at wavelength of 490 under a microplate reader (Applied Biosystems).

\section{Transwell assay}

The migrant and invasive capabilities of GC cells were measured using 24-well transwell chamber pro-adhered without Matrigel (Becton-Dickinson, San Jose, CA, USA) or with Matrigel. HGC-27 and AGS cells $\left(5 \times 10^{4}\right)$ were re-suspended in $200 \mu \mathrm{L}$ of free-serum medium and then added into the upper chamber. After culturing for $24 \mathrm{~h}$, the migrated and invaded cells on the lower chamber were fixed with $4 \%$ formaldehyde and then stained by violet crystalline for $15 \mathrm{~min}$. Subsequently, each well of transwell was observed and photographed using a microscope (Olympus, Tokyo, Japan).

\section{Analyses of glutamine, glutamate, and a-ketoglutarate (a-KG) levels}

Transfected or non-transfected HGC-27 and AGS $\left(1 \times 10^{4} /\right.$ well $)$ were seeded into 6 -well plates and cultured for $24 \mathrm{~h}$. Glutamine/Glutamate Determination Kit (Sigma-Aldrich, Merck KGaA, Darmstadt, Germany) was used for detection of concentrations of glutamine and glutamate based on user's guideline. The relative level of $\alpha-K G$ level was assessed by the $\alpha-K G$ Assay Kit (Abcam, Cambridge, MA, USA) in compliance with the specification of manufacturer's manuals.

\section{Western blot assay}

Proteins were isolated from tissues and cell lysis from HGC-27 and AGS cells by Radio-Immunoprecipitation assay (RIPA) buffer (Beyotime). After that, $40 \mu \mathrm{g}$ of total protein was segregated by $10 \%$ sodium dodecyl sulfate polyacrylamide gel electrophoresis, and then protein blots were transferred onto nitrocellulose membranes (Beyotime). After that, membranes were shaken in 5\% skim milk solution, and then incubated with appropriate primary antibodies: anti-amino-acid transporter 2 (ASCT2; ab237704; 1:1000 dilution; Abcam), anti-glutaminase (GLS1; ab156876; 1:1000 dilution; Abcam), or anti-GAPDH (ab181602; 1:1000 dilution; Abcam). After washing, the membranes were reacted with secondary antibody (ab150077; 1:2000 dilution; Abcam). Protein bands were examined using the Western Blotting Detection Kit (Solarbio, Beijing, China) and the intensity of bands was quantified using Image Lab software 5.2 (BioRad, Hercules, CA, USA).

\section{Dual-luciferase reporter assay}

The miR-876-5p binding sites in circHIPK3 or $3^{\prime}$ UTR of PIK3R1 were predicted by bioinformatics databases starBase v2.0 (http://starbase.sysu.edu.cn/). The $3^{\prime} \mathrm{UTR}$ of PIK3R1 and circHIPK3 harboring either the miR-876-5p binding site or mutant were cloned into the pmirGLO luciferase vector (Promega, Madison, WI, USA), named as circHIPK3 WT, circHIPK3 MUT, PIK3R1 3'UTR WT, or PIK3R1 3'UTR MUT, respectively. HGC-27 and AGS cells were co-transfected with constructed vectors and miR-876-5p mimic or miR-NC. After incubation for $48 \mathrm{~h}$. 
HGC-27 and AGS cells were lysed, and luciferase activity was checked using dual-luciferase reporter assay system (Promega).

\section{In vivo experiment}

The lentiviral vectors with sh-circHIPK3 (sh-circHIPK3) or control (sh-NC) were constructed by GenePharma. A total of 12 male BALB/c nude mice were purchased from Shanghai Experimental Animal Center (4-5 weeks old; Shanghai, China) and then were divided into 2 groups (6 mice per group). Sh-circHIPK3 or sh-NC stably infected HGC-27 cells $\left(5 \times 10^{6}\right.$ cells were suspended in $200 \mu \mathrm{L}$ of FBS-free culture medium) were hypodermically inoculated left axillary region of male BALB/c nude mice. Tumor growth was measured every week based on $\mathrm{V}=1 / 2 \times$ abasedonb $^{2}$ method [length (a) and width (b) length of the tumor]. At 35 days, subcutaneous tumors were removed for weight detection and further analysis. This study was directed with approval from the Institutional Animal Care and Use Committee of China-Japan Union Hospital of Jilin University.

\section{Statistical analysis}

Statistical analyses were conducted by Student's t-test and one-way analysis of variance using the SPSS 21.0 software (IBM, Somers, NY, USA). In addition, $P$-value less than 0.05 denoted significant. All data were exhibited as mean \pm standard deviation. Pearson's correlation assay was carried out to analyze the correlations relationship between miR-876-5p and circHIPK3 or PIK3R1 expression.

\section{Results}

\section{CircHIPK3 was overexpressed in GC tissues and cells}

Initially, the results of RT-qPCR revealed that circHIPK3 was significantly upregulated in GC tissues and cells compared with neighboring normal tissues and human normal gastric epithelial cell GES-1, respectively (Fig. 1a, b). In addition, we also confirmed that circHIPK 3 was more resistance to RNase $\mathrm{R}$ than linear HIPK3 mRNA, revealing that circHIPK3 was a circular RNA to some extent (Fig. 1c, d). Therefore, the biological role of circHIPK3 was investigated in the next experiments. a

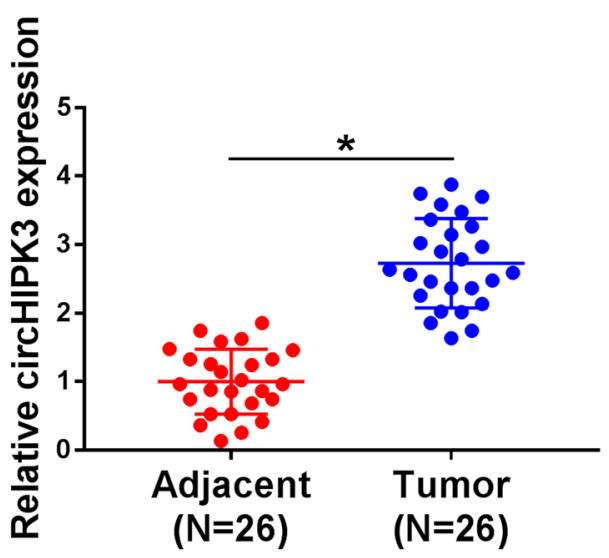

c

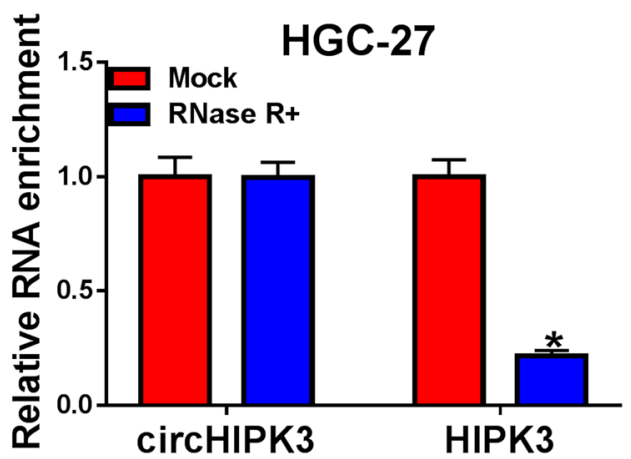

b

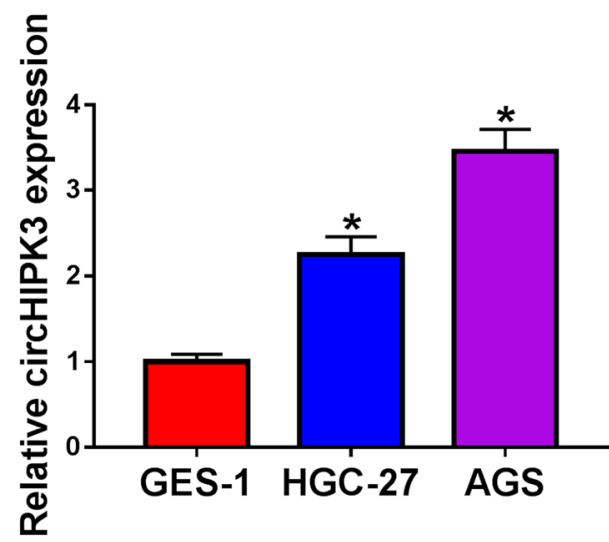

d

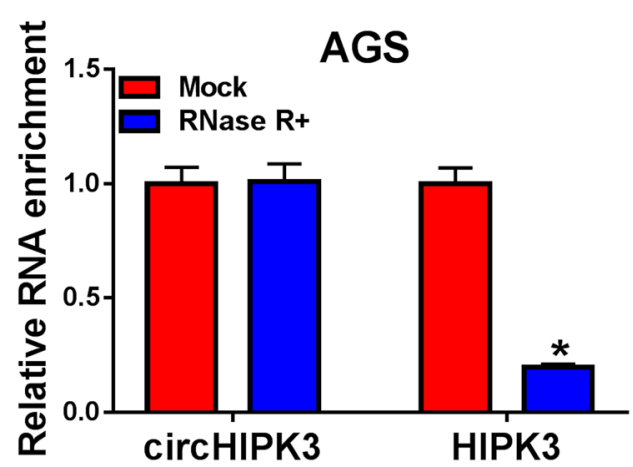

Fig. 1 The expression level of circHIPK3 in gastric cancer tissues and cells. a, $\mathbf{b}$ The relative expression level of circHIPK3 was assessed with RT-qPCR assay in gastric cancer tissues and paired neighboring normal tissues, along with GES-1, HGC-27, and AGS cells. c, d RT-qPCR was applied to determine circHIPK3 and HIPK3 mRNA levels in HGC-27 and AGS cells. ${ }^{*} P<0.05$ 
Knockdown of circHIPK3 inhibited proliferation, migration, invasion, and glutaminolysis in GC cells

As revealed in Fig. 2a, the knockdown experiments were successful using si-circHIPK3 in HGC-27 and AGS cells; the expression of circHIPK3 was decreased after transfection with si-circHIPK3. Additionally, cell viability was prominently declined at $72 \mathrm{~h}$ in $\mathrm{HGC}-27$ and AGS cells infected with si-circHIPK3 than those cells transfected with si-NC (Fig. 2b, c). By performing transwell migration and invasion assays, we found that silencing of circHIPK3 decreased migration and invasion in HGC-27 and AGS cells (Fig. 2d, e). Furthermore, the levels of glutamine, glutamate, and $\alpha-K G$ were observed to assess glutaminolysis in GC cells. As presented in Fig. $2 \mathrm{f}-\mathrm{h}$, glutamine, glutamate, and $\alpha-K G$ were all declined in HGC-27 and AGS cells by transfection with si-circHIPK3 compared with control group. Consistently, ASCT2 and GLS1 were decreased after circHIPK3 silencing in HGC27 and AGS cells (Fig. 2i-k). The above results revealed that silencing of circHIPK3 participated in proliferation, migration, invasion and glutaminolysis of GC cells, indicating the important role of circHIPK3 in GC.

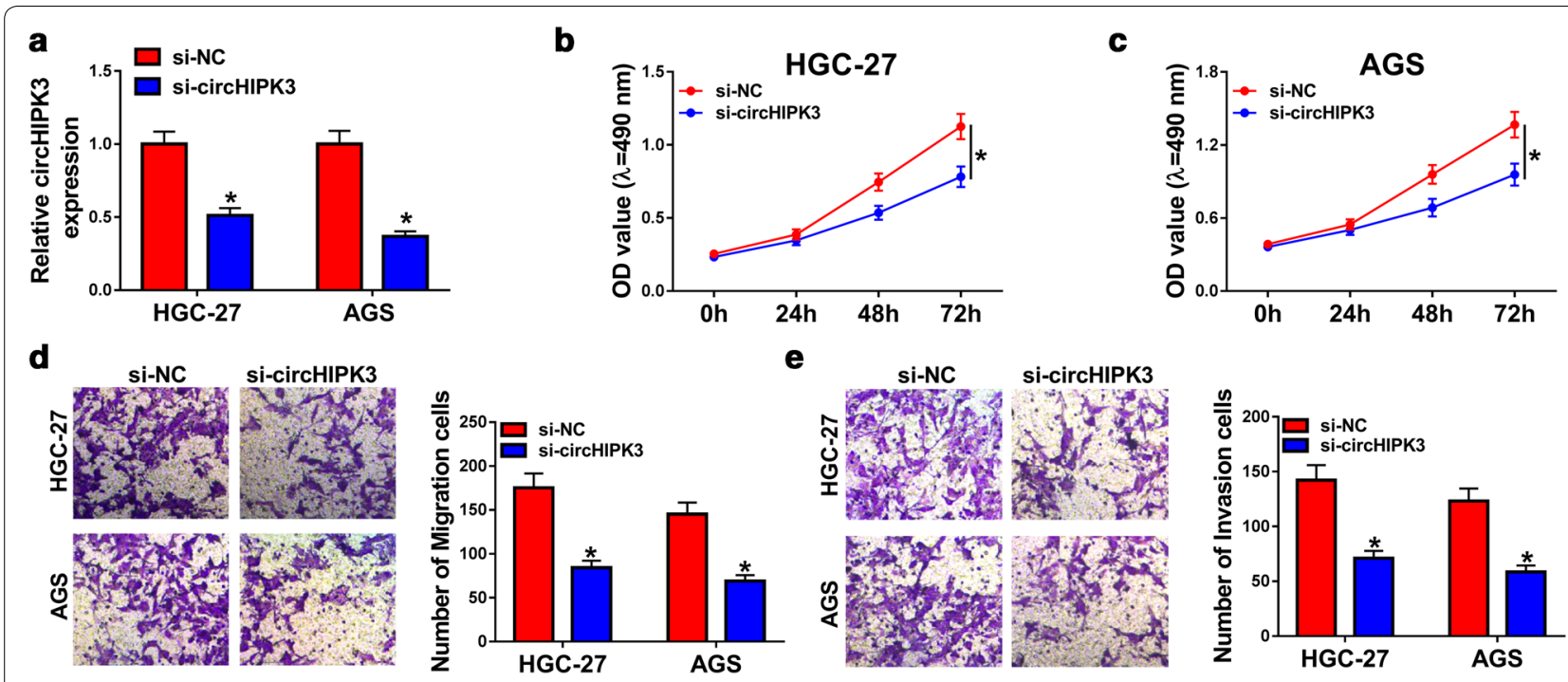

$\mathbf{f}$

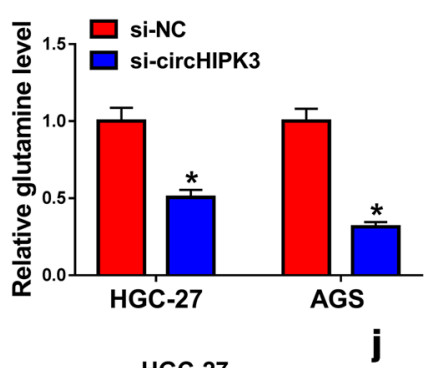

f

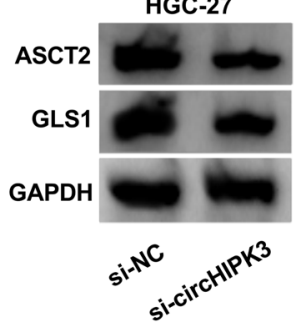

g

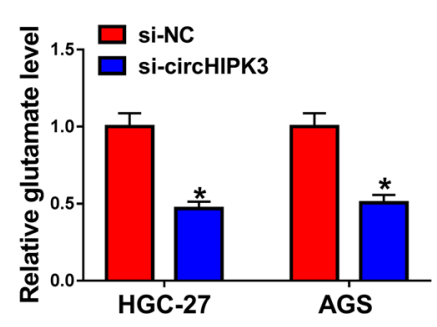

$\mathbf{k}$ h
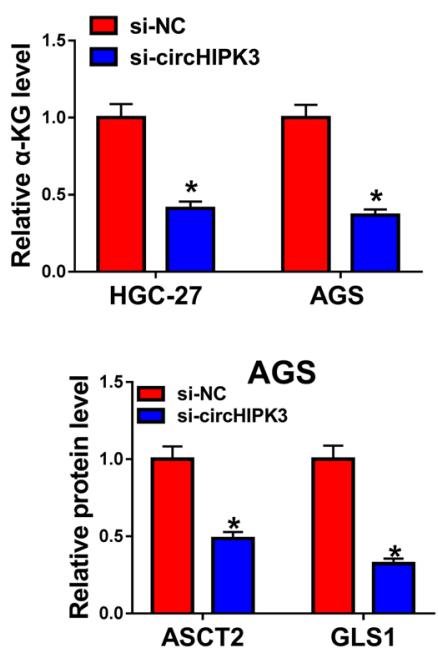

Fig. 2 Effects of circHIPK3 silencing on proliferation, migration, invasion, and glutaminolysis in gastric cancer cells. a-k HGC-27 and AGS cells were divided into two groups: si-NC and si-circHIPK3 groups. a The expression level of circHIPK3 was evaluated by RT-qPCR assay in HGC-27 and AGS cells. b, c The growth curves of HGC-27 and AGS cells were analyzed using MTT assay. d, e Transwell migration and invasion assays were performed in HGC-27 and AGS cells. $\mathbf{f}-\mathbf{h}$ The levels of glutamine, glutamate, and a-KG were displayed in HGC-27 and AGS cells. $\mathbf{i}-\mathbf{k}$ Western blot analysis was conducted to measure ASCT2 and GLS1 levels in HGC-27 and AGS cells. ${ }^{*} P<0.05$ 
MiR-876-5p was a target of circHIPK3 and was decreased in GC tissues and cells

To identify target of circHIPK3, we searched for possible target using bioinformatics software. As presented in Fig. 3a, circHIPK3 had the complementary sequence in miR-876-5p. After that, dual-luciferase reporter vectors contained wild type or mutant circHIPK3 were constructed, and HGC-27 and AGS cells were co-transfected with indicated vectors and miR-876-5p mimic or miRNC. The luciferase activity of circHIPK3 WT was inhibited in miR-876-5p mimic group, while the luciferase activity of circHIPK3 MUT had no difference between miR-876-5p and miR-NC group (Fig. 3b, c). In addition, knockdown of circHIPK3 enhanced miR-876-5p level in HGC-27 and AGS cells (Fig. 3d, e). Importantly, miR876-5p showed lower expression in GC cells and tissues with respect to GES-1 cells and adjacent normal tissues, respectively (Fig. 3f, g). An adverse connection between
circHIPK3 and miR-876-5p was observed in GC tissues (Fig. 3h). Therefore, circHIPK3 negatively regulated miR876-5p expression in GC cells.

Silencing of circHIPK3 impeded proliferation, migration, invasion and glutaminolysis of GC cells by upregulation of miR-876-5p

It has been confirmed that knockdown of circHIPK3 enhanced miR-876-5p level in HGC-27 and AGS cells, while it was abolished in HGC-27 and AGS cells by transfection with anti-miR-876-5p (Fig. 4a). Importantly, depletion of miR-876-5p abolished the inhibitory effect on proliferation in HGC-27 and AGS cells caused by si-circHIPK3 (Fig. 4b, c). Transwell analysis suggested that downregulation of miR-876-5p could rescue loss of migration and invasion capabilities caused by $\mathrm{circH}$ IPK3 knockdown (Fig. 4d, e). In addition, the levels of

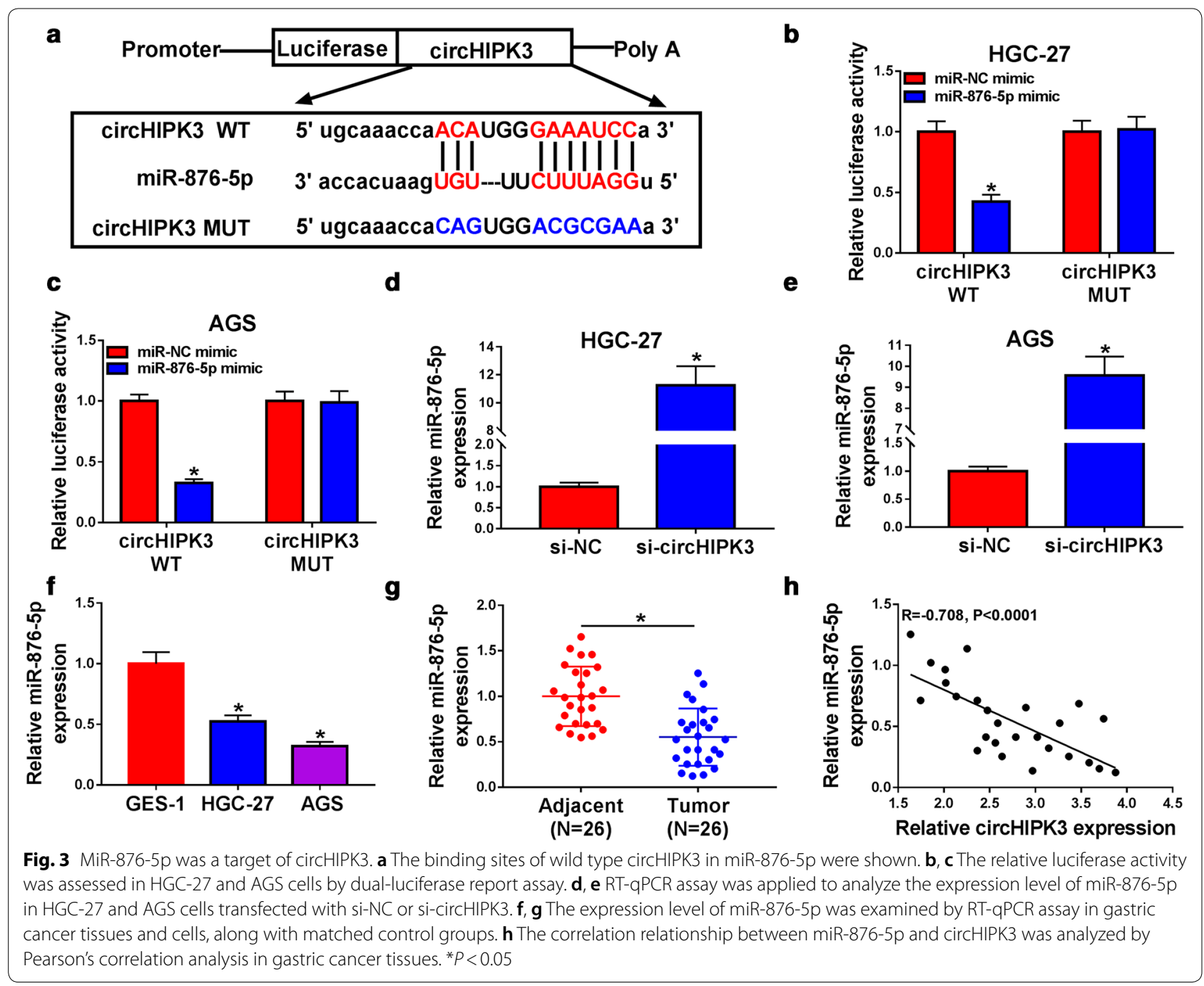




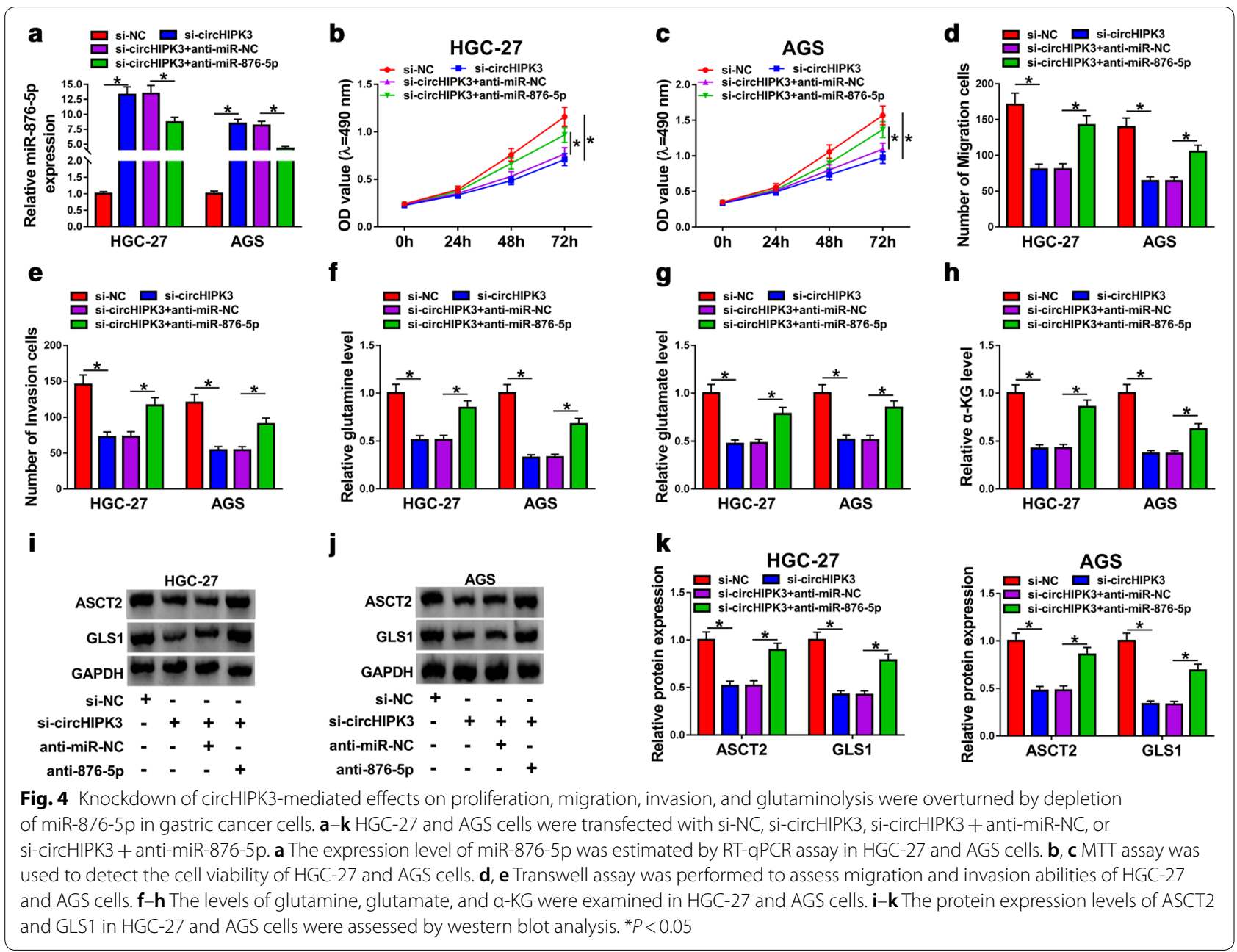

glutamine, glutamate, and $\alpha-\mathrm{KG}$ were increased in circHIPK3-silencing GC cells after knockdown of miR-876-5p (Fig. $4 \mathrm{f}-\mathrm{h}$ ). Additionally, silencing of circHIPK3 induced the reduction in ASCT2 and GLS1 expression, while cotransfection of si-circHIPK3 and anti-miR-876-5p abolished these effects (Fig. 4i-k). Collectively, circHIPK3/ miR-876-5p axis mediated proliferation, migration, invasion, and glutaminolysis of GC cells.

\section{PIK3R1 was regulated by circHIPK3/miR-876-5p axis in GC cells}

As shown in Fig. 5a, we constructed luciferase reporter vectors PIK3R1 3'UTR WT contained complementary sequence to miR-876-5p, with PIK3R1 3'UTR MUT as control. Moreover, miR-876-5p mimic declined luciferase activity of PIK3R1 3'UTR WT instead of PIK3R1 3'UTR MUT in HGC-27 and AGS cells, implying that PIK3R1 was a direct target of miR-876-5p (Fig. 5b, c). The levels of PIK3R1 were decreased in HGC-27 and AGS cells after transfection with miR-876-5p, including mRNA and protein expression (Fig. $5 \mathrm{~d}-\mathrm{f}$ ). What's more, transfection with anti-miR-876-5p overturned the decrease in PIK3R1 expression in HGC-27 and AGS cells caused by silencing of circHIPK3 (Fig. $5 \mathrm{~g}-\mathrm{i}$ ). We also confirmed that mRNA and protein expression levels of PIK3R1 were upregulated in GC tissues and cells than that in matched controls (Fig. 5j-m). Besides, PIK3R1 was observed to be negatively correlated with miR-876-5p, while positively correlated circHIPK3 expression in GC tissues (Fig. 5n, o). In summary, these results revealed that circHIPK3 regulated PIK3R1 by sponging miR-876-5p in GC.

Overexpression of PIK3R1 overturned the inhibitory effects on proliferation, migration, invasion, and glutaminolysis of GC cells caused by overexpression of miR-876-5p

The results of western blot assay indicated that the upregulation of miR-876-5p inhibited PIK3R1 expression, which was reversed by transfection with PIK3R1 (Fig. 6a). 


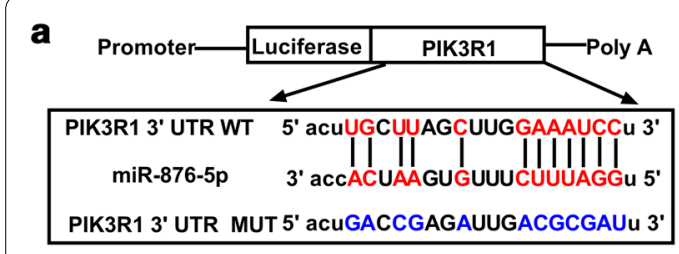

d

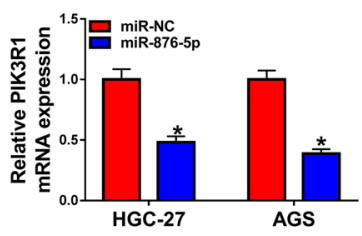

h

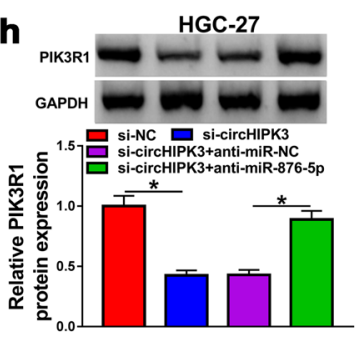

I

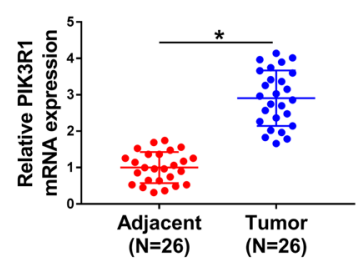

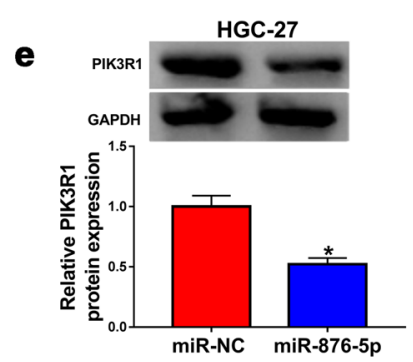

i

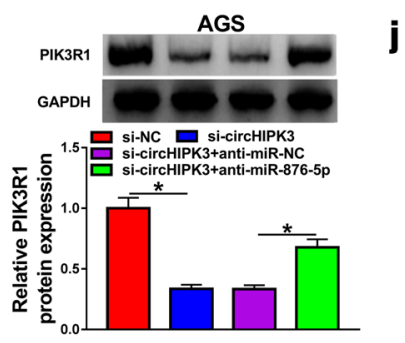

m

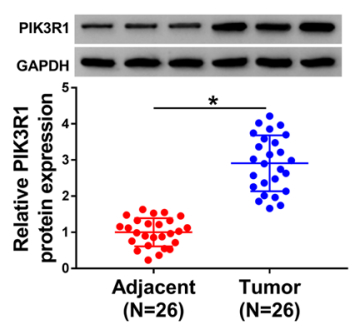

b
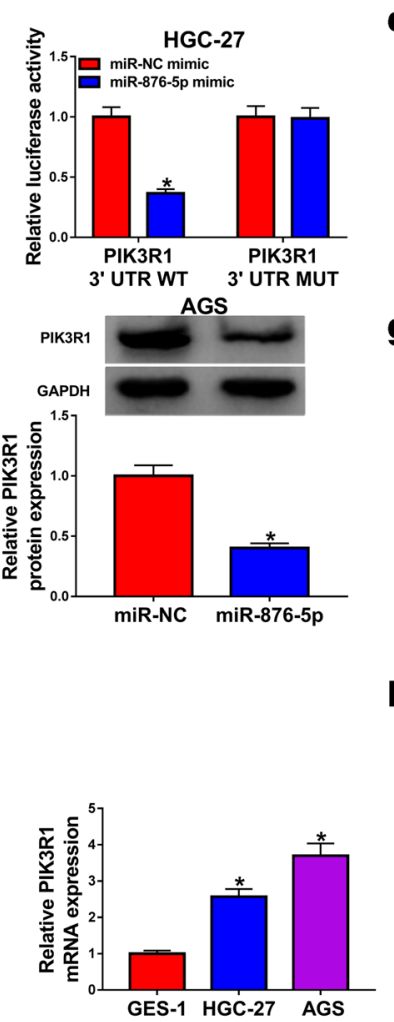

n

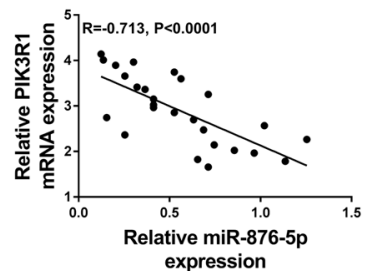

c

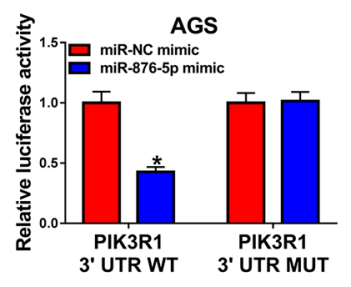

g

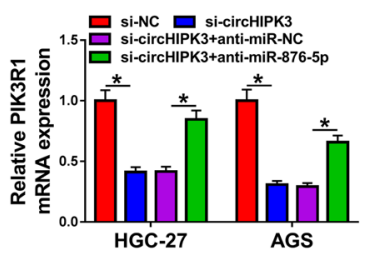

k

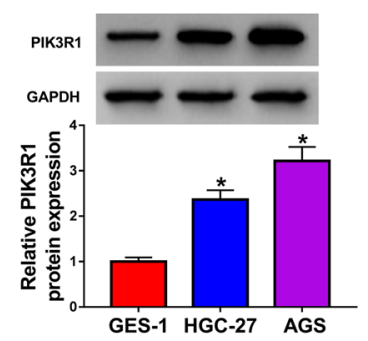

0

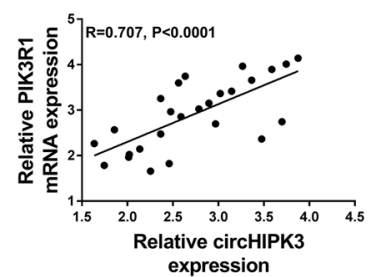

Fig. 5 CircHIPK3 regulated PIK3R1 expression by sponging miR-876-5p. a Binding regions between circHIPK3 and PIK3R1, as well as matched mutation form of PIK3R1 were presented. b, c Dual-luciferase reporter assay was introduced to test the luciferase activity in HGC-27 and AGS cells. d-f RT-qPCR and western blot assays were applied to measure PIK3R1 levels in HGC-27 and AGS cells transfected with miR-NC or miR-876-5p. g-i The mRNA and protein expression levels of PIK3R1 were assessed by RT-qPCR and western blot assays in HGC-27 and AGS cells transfected with si-NC, si-circHIPK3, si-circHIPK3 + anti-miR-NC, or si-circHIPK3 + anti-miR-876-5p. j-m The expression level of PIK3R1 was examined by RT-qPCR and western blot assays in gastric cancer tissues and cells. $\mathbf{n}-\mathbf{o}$ The correlation relationship between PIK3R1 and miR-876-5p or circHIPK3 was assessed by Pearson's correlation analysis in gastric cancer tissues. ${ }^{*} P<0.05$

The decrease of cell proliferation in miR-876-5p-overexpression cells was abrogated by upregulation of PIK3R1 (Fig. 6b, c). Moreover, transwell analysis displayed that co-transfection with miR-876-5p and PIK3R1 rescued loss of migration and invasion abilities induced by miR876-5p (Fig. 6d, e). The overexpression of PIK3R1 could obviously increase glutamine, glutamate, and $\alpha-K G$ levels in HGC-27 and AGS cells with miR-876-5p overexpression (Fig. 6f-h). Interestingly, miR-876-5p-induced inhibitory effects on ASCT2 and GLS1 expression was abrogated by overexpression of PIK3R1 in both HGC-27 and AGS cells (Fig. 6i-k). Conclusively, our results revealed that miR-876-5p regulated growth, migration, invasion and glutaminolysis by targeting PIK3R1 in GC cells.

\section{Knockdown of circHIPK3 inhibited GC tumor growth}

To probe the effect of circHIPK3 on GC cells proliferation in vivo, a xenograft tumor model in nude mice was established. As displayed in Fig. 7a, b, downregulation of circHIPK3 led to the smaller tumor volumes and weight than volumes tissues from control group. Furthermore, 


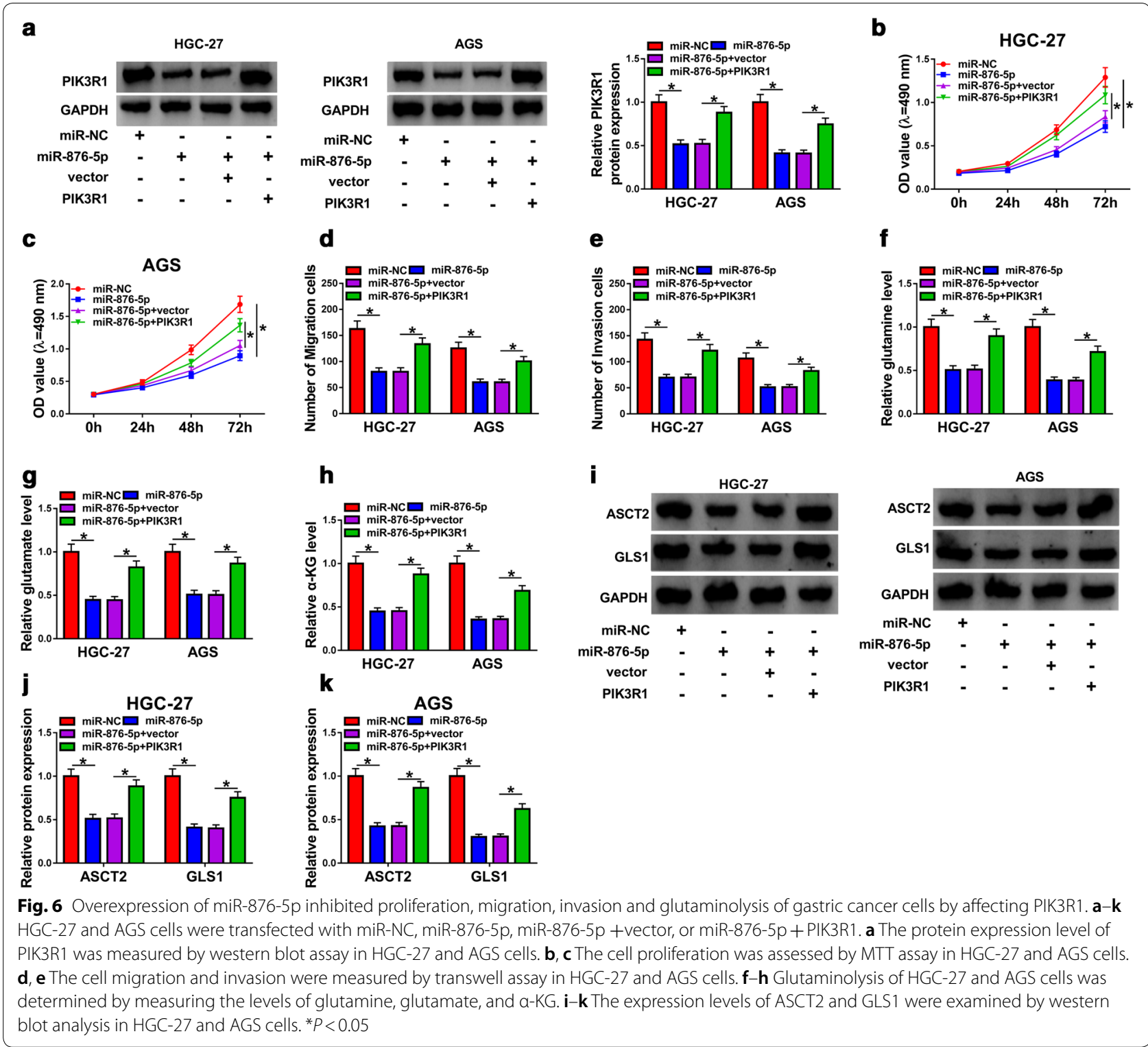

circHIPK3 was decreased, while miR-876-5p was increased in xenografts from sh-circHIPK3 group compared with sh-NC group (Fig. 7c, d). The western blot assay results indicated that suppression of circHIPK3 impeded PIK3R1 expression in xenografts (Fig. 7e). Synthetically, silencing of circHIPK3 suppressed GC growth in vivo.

\section{Discussion}

Conclusively, our study suggested the cancerogenic role of circHIPK3 in GC, and the silencing of circHIPK3 impeded GC cells proliferation, migration, invasion, as well as inhibited the tumor growth in vivo. Importantly, we found that a novel regulatory mechanism of
circHIPK3/miR-876-5p/PIK3R1 axis was involved in GC development. Furthermore, evidence has shown that survive and proliferation of cancer cells were highly dependent on glutaminolysis. ASCT2, high-affinity glutamine importer, was involved in amino acid transport and metabolism in cancer [16]. Glutamine was metabolized by rate-limiting enzyme GLS1, and eventually converted to $\alpha-K G$ [17]. Therefore, the levels of ASCT2, GLS1, and $\alpha-K G$ were closely correlated with glutaminolysis. In this paper, knockdown of circHIPK3 inhibited ASCT2, GLS1, and $\alpha-K G$ levels, suggesting silencing of circHIPK3 inhibited glutaminolysis in GC cells.

Recently, multiple studies have revealed an extensive landscape of circRNAs with exquisite regulation of the 

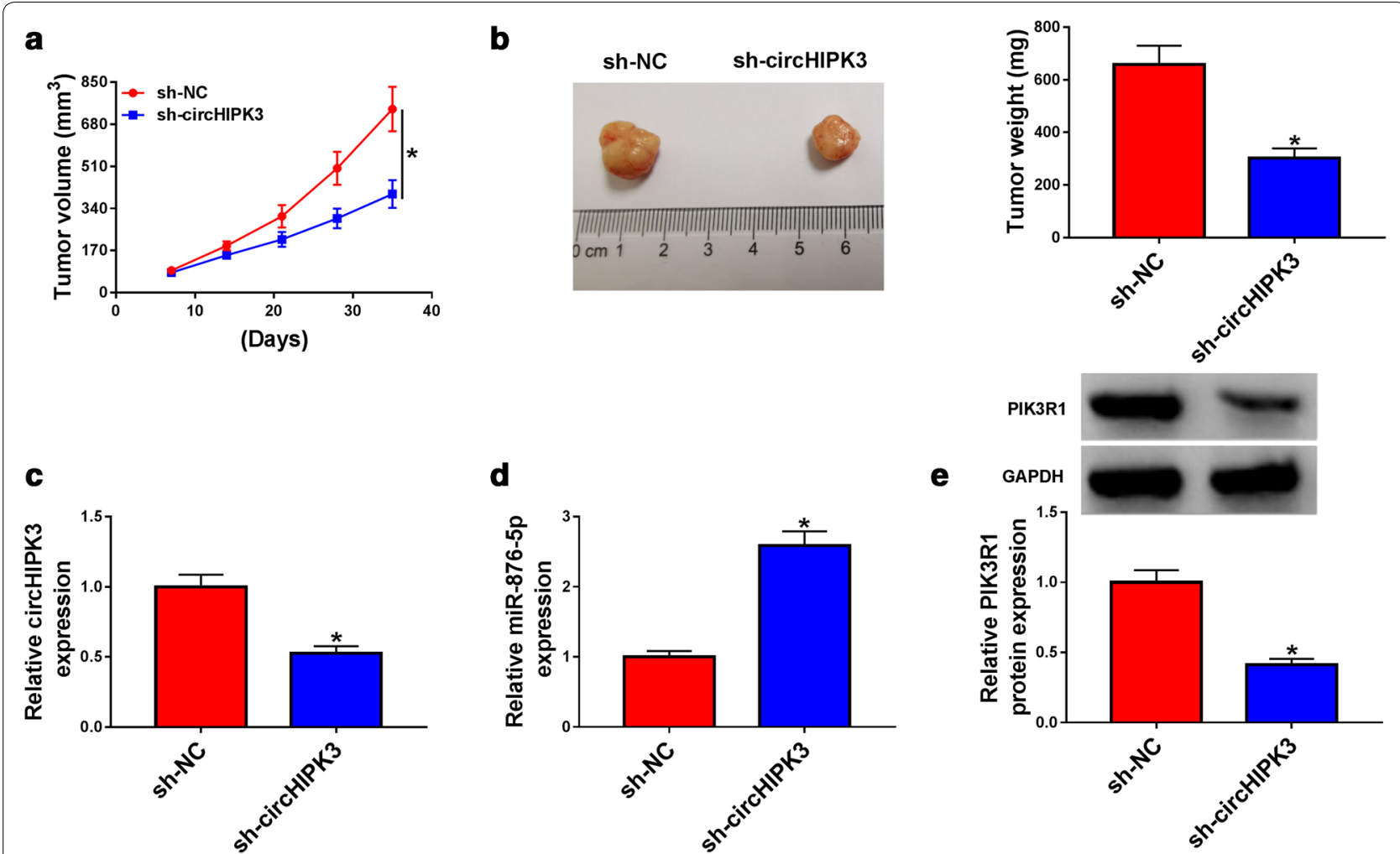

Fig. 7 Silencing of circHIPK3 impeded tumor growth in vivo. $\mathbf{a}, \mathbf{b}$ The growth curves and weight of xenograft tumors were shown. $\mathbf{c}$, d The expression levels of circHIPK3 and miR-876-5p were analyzed with RT-qPCR assay in dissected tumor tissues. e Western blot assay was used to examine the expression level of PIK3R1 in dissected tumor tissues. ${ }^{*} P<0.05$

development of malignant tumors through interaction with miRNAs $[18,19]$. The studies had shown that circHIPK3 functioned as an oncogene in malignant tumors $[6,7,20]$. By the bioinformatics prediction, we noticed that circHIPK3 had the binding sites on miR-876-5p. Subsequently, the direct association between circHIPK3 and miR-876-5p was verified using dual-luciferase reporter assay. In addition, circHIPK3 was adversely connected with miR-876-5p expression in GC tissues. As we expected, knockdown of miR-876-5p could counteract the inhibitory effects on growth, mobility, and glutaminolysis in GC cells caused by silencing of circHIPK3. Analogously, Sang et al. revealed that ciRS-7 stimulated esophageal squamous cell carcinoma progression by sponging miR-876-5p [21]. In addition, previous study by Xie et al. has unveiled the anti-tumor role of miR-876-5p in osteosarcoma by targeting c-Met [22].

For investigating the downstream target of the circHIPK3-miR-876-5p axis, dual-luciferase reporter analysis was used and identified that PIK3R1 was a functional target of miR-876-5p. The PI3K family, belong to kinases of inositol and phosphatidylinositol, were oncogenes according pervious report [23]. The regulatory subunit (p85a), the composition of PI3K, was encoded by
PIK3R1 [24], while PI3K signaling was involved in multiple human diseases, including, inflammation, malignant tumor, and diabetes $[25,26]$. We supposed that PIK3R1 was an oncogene in malignant tumors. Surely, decrease of PIK3R1 suppressed proliferation and invasion in glioblastoma multiform cells [27]. Moreover, reduction of PIK3R1 induced apoptosis and cycle arrest in colorectal cancer cells by repressed cyclin D1 expression [28]. Analogously, Huang et al. confirmed that miR-486-5p played a tumor-inhibitory role by suppressing PI3K/AKT pathway activation via targeting PIK3R1 [29]. However, the potential mechanism of PI3K/AKT regulation in GC by PIK3R1 should be further analyzed According above researches, PIK3R1 might be a disadvantageous index for prediction of GC. Not surprisingly, Xia et al. discovered PIK3R1 level was increased by nuclear paraspeckle assembly transcript 1 in GC and afterward stimulated GC progression [30]. In this study, PIK3R1 was increased in GC and adversely correlated with that of miR-876-5p expression in GC tissues. Furthermore, functional cellular experiments indicated that the upregulation of PIK3R1 increased the proliferation, migration, invasion, and glutaminolysis in miR-876-5p-overexpression GC cells. 
In the current study, circHIPK3 was upregulated in GC tissues and cells after RT-qPCR examination. The following loss-of-function assays revealed that silencing of circHIPK3 impaired proliferation, migration, invasion and glutaminolysis capacities of GC cells. Mechanistically, circHIPK3 exerted its cancerogenic functions through miR-876-5p/ PIK3R1 axis in GC.

\section{Conclusion}

In summary, circHIPK3 was upregulated in GC samples and cells when compared with adjacent noncancerous tissues and gastric epithelial cells, correspondingly. Functional experiments indicated that silencing of circHIPK3 impeded GC progression by inhibiting proliferation, migration, invasion and glutaminolysis of GC cells through miR-876-5p/PIK3R1 axis, suggesting a potential therapeutic strategy for $\mathrm{GC}$ in the future.

\section{Acknowledgements}

None.

\section{Authors' contributions}

Conception and design: QL; Development of methodology: YT; Acquisition of data: YL; Analysis and interpretation of data: $C L$ and $Q L ;$ Writing, review, and revision of article: QL; All coauthors have reviewed the article before submission. All authors read and approved the final manuscript.

\section{Funding}

None.

\section{Availability of data and materials}

All data generated or analysed during this study are included in this published article.

\section{Ethics approval and consent to participate}

All patients offered the written informed consents and the research was permitted by the Ethics Committee of China-Japan Union Hospital of Jilin University. This study was directed with approval from the Institutional Animal Care and Use Committee of China-Japan Union Hospital of Jilin University.

\section{Consent for publication}

Informed consent was obtained from all patients.

\section{Competing interests}

The authors declare that they have no competing interests.

\section{Author details}

${ }^{1}$ Department of Gastrointestinal Colorectal and Anal Surgery, China-Japan Union Hospital of Jilin University, No. 126, Xiantai Street, Changchun 130031, Jilin, China. ${ }^{2}$ Center of Physical Examination, China-Japan Union Hospital of Jilin University, Changchun 130031, Jilin, China.

Received: 1 April 2020 Accepted: 25 July 2020

Published online: 13 August 2020

\section{References}

1. Hamashima C. Current issues and future perspectives of gastric cancer screening. World J Gastroenterol WJG. 2014;20(38):13767.

2. Bray F, Ferlay J, Soerjomataram I, Siegel RL, Torre LA, Jemal A. Global cancer statistics 2018: GLOBOCAN estimates of incidence and mortality worldwide for 36 cancers in 185 countries. CA Cancer J Clin. 2018:68(6):394-424.
3. Wilusz JE, Sharp PA. A circuitous route to noncoding RNA. Science. 2013:340(6131):440-1.

4. Jens M. Circular RNAs are a large class of animal RNAs with regulatory potency. Dissecting regulatory interactions of RNA and protein. Springer; 2014. p. 69-80

5. Rong D, Lu C, Zhang B, Fu K, Zhao S, Tang W, Cao H. CircPSMC3 suppresses the proliferation and metastasis of gastric cancer by acting as a competitive endogenous RNA through sponging miR-296-5p. Mol Cancer. 2019;18(1):25

6. Zeng K, Chen X, Xu M, Liu X, Hu X, Xu T, Sun H, Pan Y, He B, Wang S. CircHIPK3 promotes colorectal cancer growth and metastasis by sponging miR-7. Cell Death Dis. 2018;9(4):417.

7. Liu N, Zhang J, Zhang L, Wang L. CircHIPK3 is upregulated and predicts a poor prognosis in epithelial ovarian cancer. Eur Rev Med Pharmacol Sci. 2018;22(12):3713-8

8. Yin Y, Song M, Gu B, Qi X, Hu Y, Feng Y, Liu H, Zhou L, Bian Z, Zhang J. Systematic analysis of key miRNAs and related signaling pathways in colorectal tumorigenesis. Gene. 2016:578(2):177-84.

9. Riquelme I, Letelier P, Riffo-Campos AL, Brebi P, Roa JC. Emerging role of miRNAs in the drug resistance of gastric cancer. Int J Mol Sci. 2016;17(3):424

10. Paladini L, Fabris L, Bottai G, Raschioni C, Calin GA, Santarpia L. Targeting microRNAs as key modulators of tumor immune response. J Exp Clin Cancer Res. 2016:35(1):103.

11. Pu M, Chen J, Tao Z, Miao L, Qi X, Wang Y, Ren J. Regulatory network of miRNA on its target: coordination between transcriptional and post-transcriptional regulation of gene expression. Cell Mol Life Sci. 2019;76(3):441-51.

12. Bao L, LV L, Feng J, Chen Y, Wang X, Han S, Zhao H. MiR-876-5p suppresses epithelial-mesenchymal transition of lung cancer by directly downregulating bone morphogenetic protein 4. J Biosci. 2017:42(4):671-81.

13. Wang Y, Xie Y, Li X, Lin J, Zhang S, Li Z, Huo L, Gong R. MiR-876-5p acts as an inhibitor in hepatocellular carcinoma progression by targeting DNMT3A. Pathol Res Pract. 2018;214(7):1024-30.

14. Xu Z, Yu Z, Tan Q, Wei C, Tang Q, Wang L, Hong Y. MiR-876-5p regulates gastric cancer cell proliferation, apoptosis and migration through targeting WNT5A and MITF. Biosci Rep. 2019. https://doi.org/10.1042/bsr20 190066.

15. Hamidi A, Song J, Thakur N, Itoh S, Marcusson A, Bergh A, Heldin C-H, Landström M. TGF- $\beta$ promotes PI3K-AKT signaling and prostate cancer cell migration through the TRAF6-mediated ubiquitylation of p85a. Sci Signal. 2017;10(486):eaal4186.

16. Van Geldermalsen M, Wang Q, Nagarajah R, Marshall A, Thoeng A, Gao D, Ritchie W, Feng Y, Bailey C, Deng N. ASCT2/SLC1A5 controls glutamine uptake and tumour growth in triple-negative basal-like breast cancer. Oncogene. 2016;35(24):3201-8.

17. Jin L, Alesi G, Kang S. Glutaminolysis as a target for cancer therapy. Oncogene. 2016;35(28):3619.

18. Li P, Yang X, Yuan W, Yang C, Zhang X, Han J, Wang J, Deng X, Yang H, Li $P$. CircRNA-Cdr1as exerts anti-oncogenic functions in bladder Cancer by sponging MicroRNA-135a. Cell Physiol Biochem. 2018:46(4):1606-16.

19. Yuan Y, Liu W, Zhang Y, Zhang Y, Sun S. CircRNA circ_0026344 as a prognostic biomarker suppresses colorectal cancer progression via microRNA-21 and microRNA-31. Biochem Biophys Res Commun. 2018;503(2):870-5.

20. Chen G, Shi Y, Liu M, Sun J. circHIPK3 regulates cell proliferation and migration by sponging miR-124 and regulating AQP3 expression in hepatocellular carcinoma. Cell Death Dis. 2018;9(2):175.

21. Sang M, Meng L, Sang Y, Liu S, Ding P, Ju Y, Liu F, Gu L, Lian Y, Li J. Circular RNA ciRS-7 accelerates ESCC progression through acting as a miR876-5p sponge to enhance MAGE-A family expression. Cancer Lett. 2018:426:37-46.

22. Xie W, Xiao J, Wang T, Zhang D, Li Z. MicroRNA-876-5p inhibits cell proliferation, migration and invasion by targeting c-Met in osteosarcoma. J Cell Mol Med. 2019;23(5):3293-301.

23. Samuels Y, Ericson K. Oncogenic PI3K and its role in cancer. Curr Opin Oncol. 2006;18(1):77-82.

24. Xinran L, Mak VC, Zhou Y, Lu Y, Cheung AN, Mills GB, Cheung LW. PIK3R1 loss activates AKT and STAT3 signaling in ovarian cancer. Philadelphia: AACR; 2018. 
25. Rathinaswamy MK, Burke JE. Class I phosphoinositide 3-kinase (PI3K) regulatory subunits and their roles in signaling and disease. Adv Biol Regul. 2019;75:100657.

26. Wong K-K, Engelman JA, Cantley LC. Targeting the PI3K signaling pathway in cancer. Curr Opin Genet Dev. 2010;20(1):87-90.

27. Weber GL, Parat M-O, Binder ZA, Gallia GL, Riggins GJ. Abrogation of PIK3CA or PIK3R1 reduces proliferation, migration, and invasion in glioblastoma multiforme cells. Oncotarget. 2011;2(11):833.

28. Sun Y, Zhao S, Tian H, Xie X, Xiao F, Li K, Song Y. Depletion of PI3K p85a induces cell cycle arrest and apoptosis in colorectal cancer cells. Oncol Rep. 2009;22(6):1435-41.
29. Huang XP, Hou J, Shen XY, Huang CY, Zhang XH, Xie YA, Luo XL. Micro RNA-486-5p, which is downregulated in hepatocellular carcinoma, suppresses tumor growth by targeting PIK3R1. FEBS J. 2015;282(3):579-94.

30. Xia T, Chen J, Wu K, Zhang J, Yan Q. Long noncoding RNA NEAT1

promotes the growth of gastric cancer cells by regulating miR-497-5p/ PIK3R1 axis. Eur Rev Med Pharmacol Sci. 2019;23(16):6914-26.

\section{Publisher's Note}

Springer Nature remains neutral with regard to jurisdictional claims in published maps and institutional affiliations.
Ready to submit your research? Choose BMC and benefit from:

- fast, convenient online submission

- thorough peer review by experienced researchers in your field

- rapid publication on acceptance

- support for research data, including large and complex data types

- gold Open Access which fosters wider collaboration and increased citations

- maximum visibility for your research: over $100 \mathrm{M}$ website views per year

At BMC, research is always in progress.

Learn more biomedcentral.com/submissions 